\title{
INSTANTONS AND BARYON DYNAMICS
}

\author{
DMITRI DIAKONOV \\ NORDITA, Blegdamsvej 17, DK-2100 Copenhagen, Denmark \\ E-mail: diakonov@nordita.dk \\ PNPI, Gatchina, St. Petersburg 188 300, Russia
}

I explain how instantons break chiral symmetry and how do they bind quarks in baryons. The confining potential is possibly irrelevant for that task.

\section{Introduction}

According to common wisdom, moving a quark away from a diquark system in a baryon generates a string, also called a flux tube, whose energy rises linearly with the separation. The string energy, however, exceeds the pion mass $m_{\pi}=140 \mathrm{MeV}$ at a modest separation of about $0.26 \mathrm{fm}$, see Fig. 1. At larger separations the would-be linear potential is screened since it is energetically favorable to tear the string and produce a pion. Virtually, the linear potential can stretch to as much as $0.4 \mathrm{fm}$ where its energy exceeds $2 m_{\pi}$ but that can happen only for a short time of $1 / m_{\pi}$. Meanwhile, the ground-state baryons are stable, and their sizes are about $1 \mathrm{fm}$. The pion-nucleon coupling is huge, and there seems to be no suppression of the string breaking by pions. The paradox is that the linear potential of the pure glue world, important as it might be to explain why quarks are not observed as a matter of principle, can hardly have a direct impact on the properties of lightest hadrons. What, then, determines their structure?

We know that, were the chiral symmetry of QCD unbroken, the lightest hadrons would appear in parity doublets. The large actual splitting between, say, $N\left(\frac{1}{2}^{-}, 1535\right)$ and $N\left(\frac{1}{2}^{+}, 940\right)$ implies that chiral symmetry is spontaneously broken as characterized by the nonzero quark condensate $\langle\bar{q} q\rangle \simeq$ $-(250 \mathrm{MeV})^{3}$. Equivalently, it means that nearly massless ('current') quarks obtain a sizable non-slash term in the propagator, called the dynamical or constituent mass $M(p)$, with $M(0) \simeq 350 \mathrm{MeV}$. The $\rho$-meson has roughly twice and nucleon thrice this mass, i.e. are relatively loosely bound. The pion is a (pseudo) Goldstone boson and is very light. The sizes of these hadrons are typically $\sim 1 / M(0)$ whereas the size of constituent quarks is given by the slope of $M(p)$ and is much less. It explains, at least on the qualitative level, why constituent quark models are so phenomenologically successful.

We see thus that the spontaneous chiral symmetry breaking (SCSB) rather 

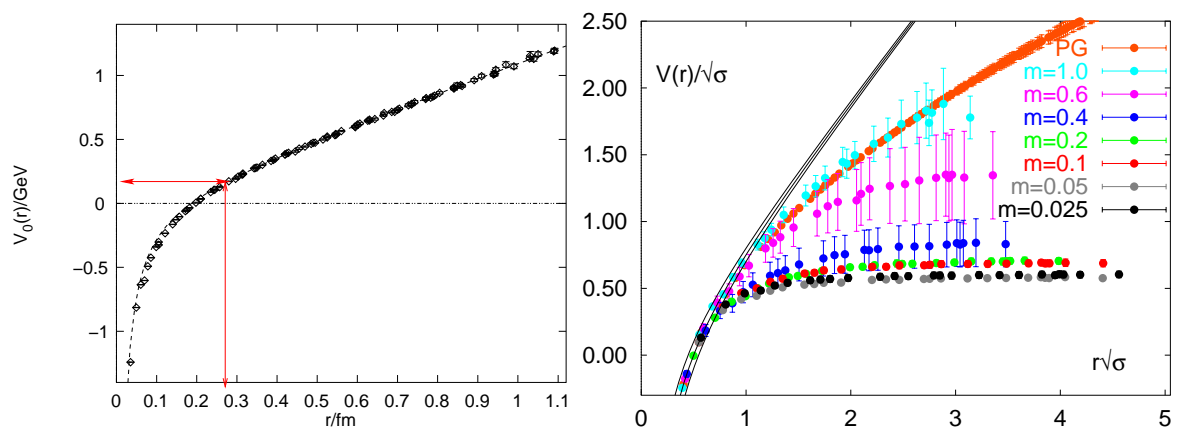

Figure 1: The lattice-simulated potential between static quarks ${ }^{1}$ exceeds $m_{\pi}$ at the separation of $0.26 \mathrm{fm}$ (left). The screening of the linear potential is clearly seen in simulations at high temperatures but below the phase transition ${ }^{2}$ (right). As one lowers the pion mass the string breaking happens at smaller distances; the scale is $\sqrt{\sigma} \simeq 425 \mathrm{MeV} \simeq(0.47 \mathrm{fm})^{-1}$.

than the expected linear confining potential of the pure glue world is key to the understanding the origin of the ground-state hadrons. It may be that for highly excited hadrons the importance of confinement forces vs SCSB is reversed: I discuss it at the end of the paper. In the main part I briefly review the instanton mechanism of the SCSB suggested and worked out by Victor Petrov and myself in the middle of the 80's. 3. - Much analytical and numerical work calculating hadron observables has supported this mechanism, see Refs. 10 for reviews. There is also growing support from direct lattice simulations, see below. Instantons induce strong interaction between quarks, leading to bound-state baryons with calculable and reasonable properties.

\section{What are instantons?}

Being a quantum field theory QCD deals with the fluctuating gluon and quark fields. A fundamental fact $\mathrm{O}$ is that the potential energy of the gluon field is a periodic function in one particular direction in the infinite-dimensions functional space; in all other directions the potential energy is oscillator-like. This is illustrated in Fig. 2.

Instanton is a large fluctuation of the gluon field in imaginary (or Euclidean) time corresponding to quantum tunneling from one minimum of the potential energy to the neighbor one. Mathematically, it was discovered by Belavin, Polyakoy Schwarz and Tiupkin; 1 the tunneling interpretation was given by V. Gribov. 0 The name 'instanton' has been introduced by 't Hooft 10 who studied many of the key properties of those fluctuations. Anti-instantons are similar fluctuations but tunneling in the opposite direction in Fig. 2. 


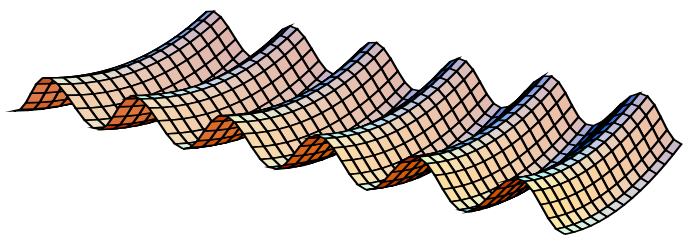

Figure 2: Potential energy of the gluon field is periodic in one direction and oscillator-like in all other directions in functional space.

Instanton fluctuations are characterized by their position in space-time $z_{\mu}$, the spatial size $\rho$ and orientation in color space $O$, all in all by 12 collective coordinates. The probability for the instanton fluctuation is, roughly, given by the WKB tunneling amplitude,

$$
\exp (- \text { Action })=\exp \left(-\frac{1}{4 g^{2}} \int d^{4} x F_{\mu \nu}^{2}\right)=\exp \left(-\frac{8 \pi^{2}}{g^{2}}\right) .
$$

It is non-analytic in the gauge coupling constant and hence instantons are missed in all orders of the perturbation theory. However, it is not a reason to ignore tunneling. For example, tunneling of electrons from one atom to another in a metal is also a nonperturbative effect but we would get nowhere in understanding metals had we ignored it. Indeed, instantons are clearly seen in nonperturbative lattice simulations of the gluon vacuum. In the upper part of Fig. 3 (taken from the paper by J. Negele et al.11) a typical snapshot of gluon fluctuations in the vacuum is shown. Naturally, it is heavily dominated by normal perturbative UV-divergent zero-point oscillations of the field. However, after smearing out these oscillations (there are now several techniques developed how to do it) one reveals a smooth background field which has proven to be nothing but an ensemble of instantons and anti-instantons with random positions and sizes. The lower part of Fig. 3 is what is left of the upper part after smoothing.

The average size of instantons found in ref. 11 is $\bar{\rho} \approx 0.36 \mathrm{fm}$ and their average separation is $\bar{R}=(N / V)^{-\frac{1}{4}} \approx 0.89 \mathrm{fm}$. Similar results have been obtained by other lattice groups using various techniques. A decade earlier the basic characteristics of the instanton ensemble were obtained analytically from the Feynman variational principle 12.13 and expressed through the only dimensional parameter $\Lambda$ one has in $\mathrm{QCD}: \bar{\rho} \approx 0.48 / \Lambda_{\overline{\mathrm{MS}}} \simeq 0.35 \mathrm{fm}, \bar{R} \approx$ $1.35 / \Lambda_{\overline{\mathrm{MS}}} \simeq 0.95 \mathrm{fm}$, if one uses $\Lambda_{\overline{\mathrm{MS}}}=280 \mathrm{MeV}$ as it follows from the DIS data. 

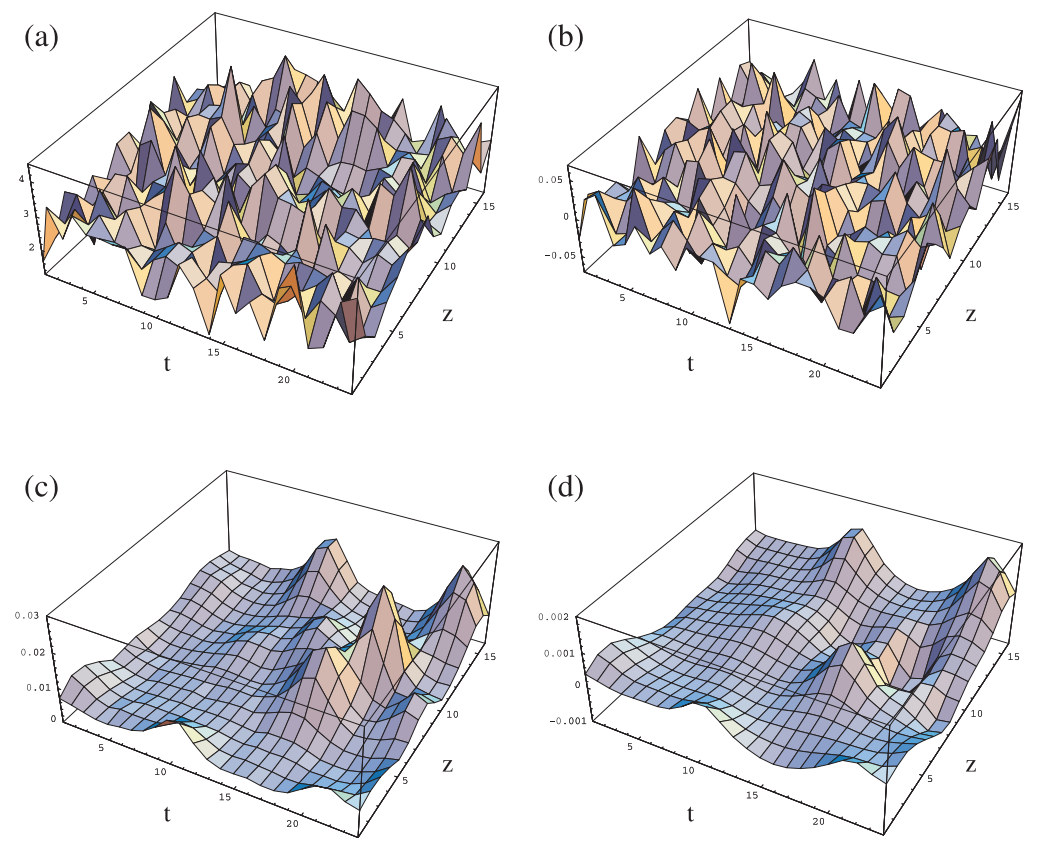

Figure 3: Smoothing out the normal zero-point oscillations reveals large fluctuations of the gluon field, which are nothing but instantons and anti-instantons with random positions and sizes. The left column shows the action density and the right column shows the topological charge density for the same snapshot. ${ }^{11}$

The theory of the instanton vacuum is based on the assumption that the QCD 'partition function' is saturated by large nonperturbative fluctuations of the gluon field (instantons), plus perturbative oscillations about them. It takes the form of a partition function of a liquid (like $\mathrm{H}_{2} \mathrm{O}$ ) of $N_{+}$instantons and $N_{-}$anti-instantons:

$$
\mathcal{Z}=\sum_{N_{ \pm}} \frac{1}{N_{+} ! N_{-} !} \prod_{I, \bar{I}} \int d^{4} z d^{7} O \frac{d \rho}{\rho^{5}}(\rho \Lambda)^{11} e^{-U_{\text {int }}}
$$

where $U_{\text {int }}$ is the interaction depending on relative positions, sizes and orientations of instantons. Actually, it is a grand canonical ensemble of interacting 'particles' since their numbers $N_{ \pm}$are not fixed but must be found from the minimum of the free energy and ultimately expressed through $\Lambda=$ 
$\frac{1}{a} \exp \left(-\frac{8 \pi^{2}}{11 g^{2}}\right)$ appearing through the 'transmutation of dimensions' from integrating over perturbative gluons ( $a$ is the UV cutoff, e.g. the lattice spacing). The qumbers for $\bar{\rho}$ and $\bar{R}$ quoted above come from the study of this ensemble. 1213 a

\section{How do instantons break chiral symmetry?}

We now switch in light quarks into the random instanton ensemble. The basic property is that massless quarks are bound by instantons with exactly zero 'energy'. These localized states are called quark zero modes, discovered by 't Hooft 10 . They have definite helicity or chirality: left-handed quarks are localized on instantons $(I)$ and right-handed are on anti-instantons $(\bar{I})$.

However, this is correct only for a single (anti)instanton. If there is a $I \bar{I}$ pair, no matter how far apart, the degeneracy of the two would-be exactly zero modes is lifted owing to the overlap of their wave functions. If there are infinitely many $I$ 's and $\bar{I}$ 's, each of them brings in a would-be zero mode but, because of the quantum-mechanical overlap, the degenerate levels split and form a continuous spectrum, meaning the delocalization of the would-be zero modes. 3 The effect is similar to the so-called Anderson conductivity: the appearance of the conductivity of electrons bound by random impurities.

It can be shown mathematically that a finite density of quark states at zero 'energy' means spontaneous chiral symmetry breaking, and one can calculate the chiral condensate from the average overlap of the zero-mode wave functions and express it through the basic quantities characterizing the instanton ensemble, i.e. the average size of instantons and their density 3 . However, there is a simpler physical argument. Each time a quark 'hops' from one random instanton to another it has to change its helicity. Delocalization implies quarks make an infinite number of such jumps. An infinite number of helicityflip transitions generates a non-slash term in the quark propagator, i.e. the dynamically-generated mass $M(p)$, see Fig. 4. It implies the spontaneous chiral symmetry breaking.

Two different formalisms have been developed in the 80's to calculate hadron observables: $(i)$ first computing an observable and then averaging it over the instanton ensemble 3 and $(i i)$ first averaging over the ensemble which leads to an effective low-energy theory, and then computing observables in the effective theory. 4 Despite very different appearance the two formalisms give

${ }^{a}$ The first study of the instanton ensemble on qualitative level was performed by Gallan, Dashen and Gross 14 and later on by Shuryak 15 . Ilgenfritz and Mueller-Preussker 16 were the first to study the instanton ensemble quantitatively by modelling the interactions by a hard-core repulsion. 


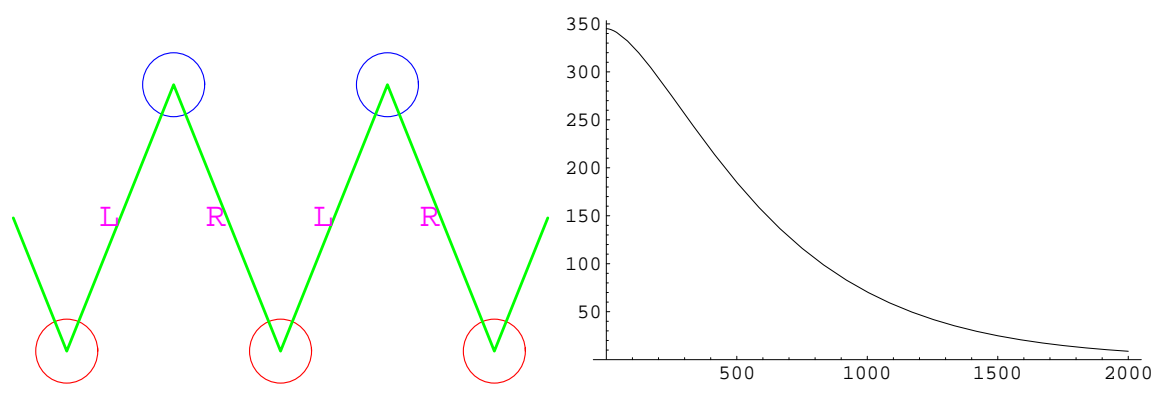

Figure 4: Quarks hopping from instantons to anti-instantons and vice versa flip helicity (left). An infinite number of such jumps generates a dynamical mass $M(p)$, in $\mathrm{MeV}$ (right). ${ }^{3}$

identical final results. Let me list a few of them:

$$
\begin{aligned}
& <\bar{q} q>=-\frac{\text { const. }}{\bar{R}^{2} \bar{\rho}} \simeq-(255 \mathrm{MeV})^{3}, \quad M(0)=\text { const. } \frac{\pi \bar{\rho}}{\bar{R}^{2}} \simeq 345 \mathrm{MeV}, \\
& F_{\pi}=\text { const. } \frac{\bar{\rho}}{\bar{R}^{2}} \sqrt{\log \frac{\bar{R}}{\bar{\rho}}} \simeq 100 \mathrm{MeV} \quad \text { vs. } 94 \mathrm{MeV} \text { (exper) } \\
& m_{\eta^{\prime}}=\frac{\text { const. }}{\bar{\rho}} \simeq 980 \mathrm{MeV} \quad \text { vs. } \quad 958 \mathrm{MeV} \text { (exper) } \ldots
\end{aligned}
$$

where "const." are computable numerical constants of the order of unity.

Recently, the instanton mechanism of the SCSB has been scrutinized by direct lattice methods. $17.1 \mathrm{~B}, \mathrm{C}$ At present there is one group 19 challenging the instanton mechanism. However, the density of alternative 'local structures' found there explodes as the lattice spacing decreases, and this must be sorted out first. Studies by other groups 17.18 support or strongly support the mechanism described above. In particular, in a recent paper Gattringer 18 convincingly demonstrates that quarks in near-zero modes concentrate in the regions where the gluon field is either self-dual (I's) or anti-self-dual (I's). Since near-zero modes are responsible for the SCSB it is a direct confirmation of the instanton mechanism.

\section{Baryons}

There is a remarkable evidence of the importance of instantons for the baryon structure. In Ref. 11 the so-called density-density correlation function inside the nucleon has been measured both in the full vacuum and in the instanton vacuum resulting from the full one by means of smoothing. The correlation 


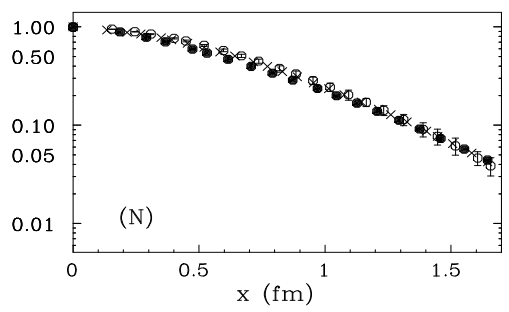

Figure 5: Density-density correlation function in the nucleon. ${ }^{11}$ Filled circles are measurements in the full gluon vacuum (corresponding to Fig. 3a,b) while open circles are measured in the vacuum with instantons only (Fig. 3c,d). Despite that linear confining potential is absent in the instanton vacuum the nucleon structure seems to be very well reproduced.

in question is between the densities of $u$ and $d$ quarks separated by a distance $x$ inside the nucleon which is created at some time and annihilated at a later time. The two correlators ('full' and 'instanton') are depicted in Fig. 5: one observes a remarkable agreement between the two, up to $x=1.7 \mathrm{fm}$.

It must be stressed that neither the one-gluon exchange nor the linear confining potential present in the full gluon vacuum survive the smoothing of the gluon field shown in Fig. 3. Nevertheless, quark correlations in the nucleon remain basically unaltered! It means that neither the one-gluon exchange nor the linear confining potential are important for the quark binding inside the nucleon. As a matter of fact, the same remark can be addressed to the lightest mesons $\pi$ and $\rho$ since the density-density correlators for these hadrons also remain basically unchanged as one goes from the full glue to the reduced instanton vacuum. 11 Therefore, one must be able to explain at least the lightest $\pi, \rho, N$ on the basis of instantons only.

The dynamics remaining in the instanton vacuum is the SCSB, the apearance of the dynamical quark mass $M(p)$, and quark interactions induced by the possibility that they scatter off the same instanton. Actualy these interactions named after 't Hooft, are quite strong. 6 . They are in fact so strong that for quark and antiquark in the pion channel they eat up the $700 \mathrm{MeV}$ of twice the constituent quark mass to nil, as required by the Goldstone theorem. In the vector meson channel 't Hooft interactions are suppressed, and that is why the $\rho$ mass is roughly twice the constituent quark mass. In the nucleon they are fully at work but in a rather peculiar way: instanton-induced interactions can be iterated as many times as one wishes in the exchanges between quarks, see Fig. 6, left. It can be easily verified that the diagram in Fig. 6, left, can be drawn as three continuous quark lines going from the l.h.s of the diagram to its r.h.s., without adding closed loops. Therefore, that kind of interaction 

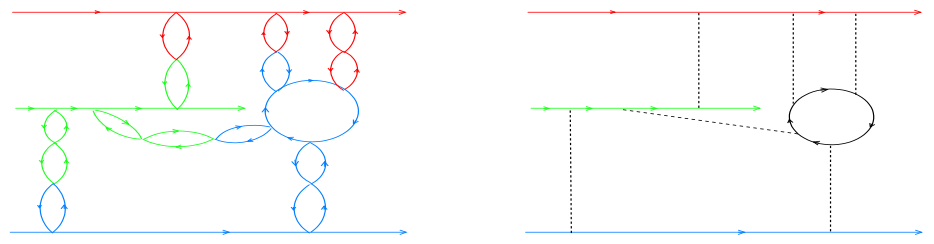

Figure 6: 't Hooft interactions in the nucleon (left) essentially come to quarks interacting via pion fields (right).

arises already in the so-called quenched approximation. At the same time, it yields plenty of Z-graphs absent in "valence QCD" but which are necessary to reproduce hadron properties. 20

Summing up all interactions of the kind shown in Fig. 6, left, seems to be a hopeless task. Nevertheless, the nucleon binding problem can be solved exactly when two simplifications are used. The first exploits the fact that in the instanton vacuum there are two lightest degrees of freedom: pions (since they are the Goldstone bosons) and quarks with the dynamical mass $M$. All the rest collective excitations of the instanton vacuum are much heavier, and one may wish to neglect them. Pions arise from summing up the $q \bar{q}$ bubbles schematically shown in Fig. 6, left. The resulting effective low-energy theory takes the form of the non-linear $\sigma$-model: 421

$$
\mathcal{L}_{\text {eff }}=\bar{q}\left[i \not \partial-M \exp \left(i \gamma_{5} \pi^{A} \tau^{A} / F_{\pi}\right)\right] q .
$$

The absence of the explicit kinetic energy term for pions (which would leag to the double counting) distinguishes it from the Manohar-Georgi model. 22 Expanding the exponent to the first power in $\pi^{A}$ we find that the dimensionless pion-constituent quark coupling,

$$
g_{\pi q q}=\frac{M}{F_{\pi}} \approx 4
$$

is quite strong. The domain of applicability of the low-energy effective theory (6) is restricted by momenta $p<1 / \bar{\rho}=600 \mathrm{MeV}$, which is the inverse size of constituent quarks. At higher momenta one starts to feel the internal structure of constituent quarks, and the two lightest degrees of freedom of Eq. (6) become insufficient. However, the expected typical momenta of quarks in the nucleon are of the order of $M \approx 345 \mathrm{MeV}$, which is inside the domain of applicability of the low-momentum effective theory. 
The chiral interactions of constituent quarks in the nucleon, following from the effective theory (5), are schematically shown in Fig. 6, right, where quarks are denoted lines with arrows. Notice that, since there is no explicit kinetic energy for pions in Eq. (6), the pion propagates only through quark loops. Quark loops induce also many-quark interactions indicated in Fig. 6 as well. We see that the emerging picture is rather far from a simple one-pion exchange between the constituent quarks: the non-linear effects in the pion field are essential.

The second simplification is achieved in the limit of large $N_{c}$. For $N_{c}$ colors the number of constituent quarks in a baryon is $N_{c}$ and all quark loop contributions are also proportional to $N_{c}$. Therefore, at large $N_{c}$ one can speak about a classical self-consistent pion field inside the nucleon: quantum fluctuations about the classical field are suppressed as $1 / N_{c}$. The problem of summing up all diagrams of the type shown in Fig. 6 is thus reduced to finding a classical pion field pulling $N_{c}$ massive quarks together to form a bound state.

\section{Chiral Quark-Soliton Model}

Let us imagine a classical time-independent pion field which is strong and spatially wide enough to form a bound-state level in the Dirac equation following from Eq. (6). The background chiral field is color-neutral, so one can put $N_{c}$ quarks on the same level in an antisymmetric state in color, i.e. in a colorsinglet state. Thus we obtain a baryon state, as compared to the vacuum.

One has to pay for the creation of this trial pion field, however. Since there are no terms depending directly on the pion field in the low-momentum theory (6) the energy of the pion field is actually encoded in the shift of the lower negative-energy Dirac sea of quarks, as compared to the free case with zero pion field. The baryon mass is the sum of the bound-state energy and of the aggregate energy of the lower Dirac sea. It is a functional of the trial pion field; one has to minimize it with respect to that field to find the self-consistent pion field that binds quarks inside a baryon. It is a clean-cut problem, and can be solved numerically or, approximately, analylically. The description of baryons based on this construction has been named the Chiral Quark-Soliton Model (CQSM).23,24,25

The model reminds the large- $Z$ Thomas-Fermi atom where $N_{c}$ plays the role of $Z$. Fortunately, corrections to the model go as $1 / N_{c}$ or even as $1 / N_{c}^{2}$ and have been computed for many observables. In the Thomas-Fermi model of atoms corrections to the self-consistent (electric) field are of the order of $1 / \sqrt{Z}$ and for that reason are large unless atoms are very heavy.

In the end of the 80's and the beginning of the 90's dozens of baryon 
characteristics have been computed in the CQSM, including masses, magnetic moments, axial constants, formfactors, splittings inside the mutliplets and between multiplets, polarizability, fraction of nucleon spin carried by quarks, etc. - see26.6 for a review and references therein. Starting from '96 a new class of problems have heen addressed, namely parton distributions in the nucleon at low virtuality. 20 Parton distributions are a snapshot of the nucleon in the infinite momentum frame. One needs an inherently relativistic model in order to describe them consistently. For example, a bag model or any other nonrelativistic model with three quarks in a bound state, being naively boosted to the infinite-momentum frame gives a negative distribution of antiquarks, which is nonsense. On the contrary, being a relativistic field-theoretic model CQSM predicts parton distributions that satisfy all general requirements known in full QCD, like positivity and sum rules constraints.

Numerous parton distributions have been computed in the CQSM, mainly by the Bochum group. 2722.29 There have been a number of mysteries from naive quark models' point of view: the large number of antiquarks already at a low virtuality, the 'spin crisis', the large flavor asymmetry of antiquarks, etc. The CQSM explains all those 'mysteries' in a natural way as it incorporates, together with valence quarks bound by the isospin-1 pion field, the negativeenergy Dirac sea. Furthermore, the CQSM predicts nontrivial phenomena that have not been observed so far: large flavor asymmetry of the polarized antiquarks 29 , transversitydictributions 30 , peculiar shapes of the so-called skewed

parton distributions 31 and other phenomena in hard exclusive reactions. 32 Baryon dynamics is rich and far from naive "three quarks" expectations.

\section{Conclusions}

1. The would-be linear confining potential of the pure glue world is necessarily screened by pion production at very moderate separations between quarks. Therefore, light hadrons should not be sensitive to confinement forces but rather to the dynamics of the spontaneous chiral symmetry breaking (SCSB).

2. Most likely, the SCSB is driven by instantons - large nonperturbative fluctuations of the gluon field having the meaning of tunneling. The SCSB is due to 'hopping' of quarks from one randomly situated instanton to another, each time flipping the helicity. The instanton theory of the SCSB is in agreement with the low-energy phenomenology ( $c f$. the chiral condensate $\langle\bar{q} q\rangle$, the dynamical quark mass $M(p), F_{\pi}, m_{\eta^{\prime}} \ldots$ ) and seems to be confirmed by direct lattice methods. Furthermore, lattice simulations indicate that instantons alone are responsible for the properties of lightest hadrons $\pi, \rho, N, \ldots$ 
3. Summing up instanton-induced quark interactions in baryons leads to the Chiral Quark-Soliton Model where baryons appear to be bound states of constituent quarks pulled together by the chiral field. The model enables one to compute numerous parton distributions, as well as 'static' characteristics of baryons - with no fitting parameters.

4. For highly excited baryons $(m=1.5-3 \mathrm{GeV})$ the relative importance of confining forces vs. those of the SCSB may be reversed. One can view a large-spin $J$ resonance as due to a short-time stretch of an unstable string or, alternatively, as a rotating elongated pion cloud. 33 What picture is more adequate is a question to experiment. In the first case the dominant decay is on the average of the type $\operatorname{Bar}_{J} \rightarrow \operatorname{Bar}_{\sim J / 2}+\mathrm{Mes}_{\sim J / 2}$; in the second case it is mainly a cascade $\operatorname{Bar}_{J} \rightarrow \operatorname{Bar}_{J-1}+\pi \rightarrow \operatorname{Bar}_{J-2}+\pi \pi \rightarrow \ldots$ Studying resonances can elucidate the relation between chiral and confining forces.

\section{References}

1. G. Bali, K. Schilling and A. Wachter, in Confinement 95, eds. H. Toki et al. (World Scientific, Singapore, 1995) p.82, hep-lat/9506017.

2. F. Karsch, E. Laermann and A. Peikert, Nucl. Phys. B605, 579 (2001), hep-lat/0012023.

3. D. Diakonov and V. Petrov, Phys. Lett. B 147, 351 (1984);

Sov. Phys. JETP 62, 204 (1985); ibid. 62, 431 (1985);

Nucl. Phys. B272, 457 (1986).

4. D. Diakonov and V. Petrov, Spontaneous Breaking of Chiral Symmetry in the Instanton Vacuum, preprint LNPI-1153 (1986), in: Hadron Matter under Extreme Conditions, eds. G. Zinoviev and V. Shelest (Naukova dumka, Kiev, 1986) p.192.

5. D. Diakonov, in: Proc. of Enrico Fermi School, Course 130, eds. A. Di Giacomo and D. Diakonov (IOS Press, 1996), hep-ph/9602375; T. Schafer and E. Shuryak, Rev. Mod. Phys. 70, 323 (1998).

6. D. Diakonov and V. Petrov, in: At the Frontier of Particle Physics, ed. M. Shifman (World Scientific, Singapore, 2001) p.359, hep-ph/0009006.

7. L.D. Faddeev, Looking for multi-dimensional solitons, in: Non-local Field Theories (Dubna, 1976) p.207; R. Jackiw and C. Rebbi, Phys. Rev. Lett. 37, 172 (1976).

8. A. Belavin, A. Polyakov, A. Schwartz and Yu. Tyupkin, Phys. Lett. 59, 85 (1975).

9. A. Polyakov, Nucl. Phys. B120, 429 (1977).

10. G. 't Hooft, Phys. Rev. D14, 3432 (1976).

11. M.C. Chu, J.M. Grandy, S. Huang and J.W. Negele, Phys. Rev. D 49, 
6039 (1994), hep-lat/9312071.

12. D. Diakonov and V. Petrov, Nucl. Phys. B245, 259 (1984).

13. D. Diakonov, M. Polyakov and C. Weiss, Nucl. Phys. B461, 539 (1996), hep-ph/9510232.

14. C. Callan, R. Dashen and D. Gross, Phys. Rev. D17, 2717 (1978).

15. E. Shuryak, Nucl. Phys. B203, 93 (1982).

16. E.M. Ilgenfritz and M. Müller-Preussker, Nucl. Phys. B184, 443 (1981).

17. J. Negele, Nucl. Phys. Proc. Suppl. 73, 92 (1999), hep-lat/9810053.

18. T. DeGrand and A. Hasenfratz, Phys. Rev. D64, 034512 (2001), hep-lat/0012021; C. Gattringer et al., Nucl. Phys. B617, 101 (2001), hep-lat/0107016; T. Blum et al., Phys. Rev. D65 014504 (2002); hep-lat/0105006; R. G. Edwards and U. M. Heller, Phys. Rev. D65, 014505 (2002); hep-lat/0105004; I. Hip et al., Phys. Rev. D65, 014506 (2002), hep-lat/0105001; C. Gattringer, hep-lat/0202002.

19. I. Horváth et al., hep-lat/0201008.

20. K.F. Liu et al., Phys. Rev. D59, 112001 (1999), hep-ph/9806491.

21. D. Diakonov, in: Advanced school on non-perturbative quantum field physics, (World Scientific, Singapore, 1998) p.1, hep-ph/9802298.

22. A. Manohar and H. Georgi, Nucl. Phys. B234, 189 (1984).

23. S. Kahana, G. Ripka and V. Soni, Nucl. Phys. A 415, 351 (1984);

S. Kahana and G. Ripka, Nucl. Phys. A 429, 462 (1984).

24. M.S. Birse and M.K. Banerjee, Phys. Lett. B 136, 284 (1984).

25. D. Diakonov and V. Petrov, Sov. Phys. JETP Lett. 43, 57 (1986); D. Diakonov, V. Petrov and P. Pobylitsa, in: Proc. 21st PNPI Winter School (Leningrad, 1986) p.158; Nucl. Phys. B 306, 809 (1988).

26. C. Christov et al., Prog.Part.Nucl.Phys. 37, 91 (1996), hep-ph/9604441.

27. D. Diakonov et al., Nucl. Phys. B 480, 341 (1996), hep-ph/9606314; Phys. Rev. D 56, 4069 (1997), hep-ph/9703420.

28. P. Pobylitsa et al., Phys. Rev. D59, 034024 (1999), hep-ph/9804436.

29. B. Dressler, K. Goeke, M. Polyakov and C. Weiss, Eur. Phys. J. C14, 147 (2000), hep-ph/9909541.

30. P. Pobylitsa and M. Polyakov, Phys. Lett. B 389, 350 (1996), hep-ph/9608434.

31. V. Petrov et al., Phys. Rev. D 57, 4325 (1998), hep-ph/9710270.

32. K. Goeke, M. Polyakov and M. Vanderhaeghen, Prog. Part. Nucl. Phys. 47, 401 (2001), hep-ph/0106012.

33. D. Diakonov and V. Petrov, Rotating chiral solitons lie on linear Regge trajectories, preprint LNPI-1394 (1988); see also D. Diakonov, Acta Phys. Polon. B25, 17 (1994). 